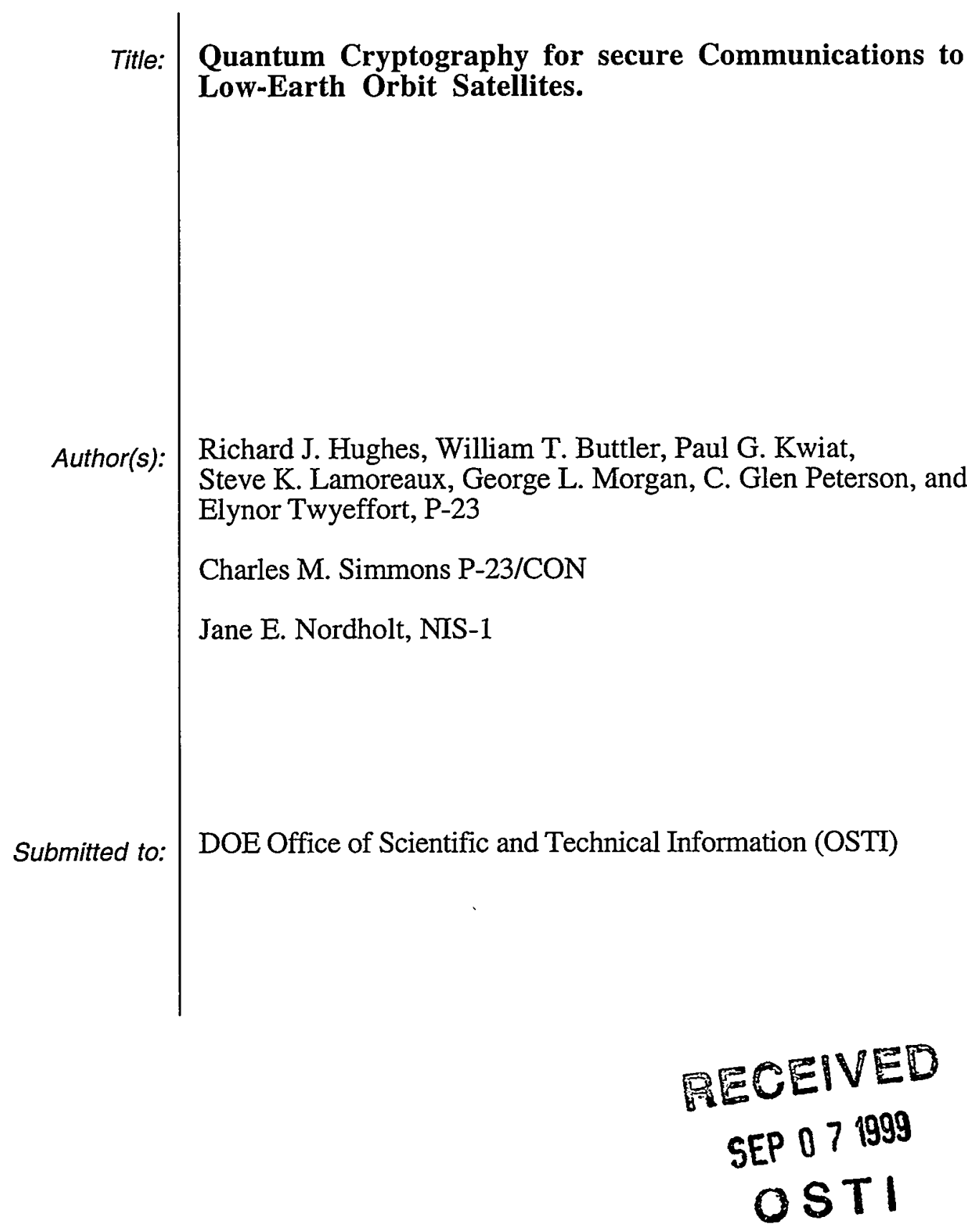

Los Alamos National Laboratory, an affirmative action/equal opportunity employer, is operated by the University of California for the U.S. Department of Energy under contract W-7405-ENG-36. By acceptance of this article, the publisher recognizes that the U.S. Govemment retains a nonexclusive, royaltyfree license to publish or reproduce the published form of this contribution, or to allow others to do so, for U.S. Government purposes. Los Alamos National Laboratory requests that the publisher identify this article as work performed under the auspices of the U.S. Department of Energy. Los Alamos National Laboratory strongly supports academic freedom and a researcher's right to publish; as an institution, however, the Laboratory does not endorse the viewpoint of a publication or guarantee its technical correctness. 


\section{DISCLAIMER}

This report was prepared as an account of work sponsored by an agency of the United States Government. Neither the United States Government nor any agency thereof, nor any of their employees, make any warranty, express or implied, or assumes any legal liability or responsibility for the accuracy, completeness, or usefulness of any information, apparatus, product, or process disclosed, or represents that its use would not infringe privately owned rights. Reference herein to any specific commercial product, process, or service by trade name, trademark, manufacturer, or otherwise does not necessarily constitute or imply its endorsement, recommendation, or favoring by the United States Government or any agency thereof. The views and opinions of authors expressed herein do not necessarily state or reflect those of the United States Government or any agency thereof. 


\section{DISCLAIMER}

\section{Portions of this document may be illegible in electronic image products. Images are produced from the best available original document.}




\title{
Quantum Cryptography for Secure Communications to Low-Earth Orbit Satellites
}

\author{
Richard J. Hughes*, William T. Buttler, Paul G. Kwiat, \\ Steve K. Lamoreaux, George L. Morgan, C. Glen Peterson, Elynor Twyeffort, P-23, \\ Charles M. Simmons P-23/CON, and Jane E. Nordholt, NIS-1
}

\begin{abstract}
This is the final report of a three-year, Laboratory Directed Research and Development (LDRD) project at Los Alamos National Laboratory (LANL). Quantum cryptography is an emerging technology in which two parties may simultaneously generate shared, secret cryptographic key material using the transmission of quantum states of light. The security of these transmissions is based on the inviolability of the laws of quantum mechanics. An adversary can neither successfully tap the quantum transmissions, nor evade detection. Key material is built up using the transmission of a single-photon per bit. We have developed an experimental quantum cryptography system based on the transmission of non-orthogonal single-photon polarization states to generate shared key material over line-of-sight optical links. Our results provide strong evidence that cryptographic key material could be generated on demand between a ground station and a satellite (or between two satellites), allowing a satellite to be securely re-keyed on orbit.
\end{abstract}

\section{Background and Research Objectives}

Satellite telemetry is becoming more susceptible to eavesdropping while the importance of satellite intelligence and the security of data and command paths to spacebased assets is continually increasing. Information security can be assured by encryption, requiring shared secret cryptographic key material (cryptovariables) for use in encryption hardware on the orbiting satellite and at ground stations. However, the secure distribution of key material to orbital assets is itself subject to increasing challenges because codebreaking techniques and algorithms are advancing rapidly.[1] If the key material supplied at launch should be used up during normal operations or compromised, an issue arises of how to securely re-key a satellite on-orbit. (For example, it is impractical to send a courier to a satellite.) If the sender ("Alice") and recipient ("Bob") communicate solely through classical messages it is impossible for them to generate a certifiably secret key owing to the possibility of passive eavesdropping by third parties ("Eve"). However, secure key generation becomes possible if they communicate with single-photon transmissions using

\footnotetext{
*Principal Investigator, e-mail: rxh@lanl.gov
} 
the emerging technology of quantum cryptography, or more accurately, quantum key distribution (QKD).[2] (A small amount of shared secret key material is required to perform initial authentication.) The aim of this project has been to develop an experimental ground-based "free-space" QKD system to validate the essential physics of surface-tosatellite QKD.

The security of QKD is based on the inviolability of the laws of quantum mechanics and provably secure (information theoretic) public discussion protocols. Eve can neither "tap" the key transmissions owing to the indivisibility of quanta[3] nor copy them faithfully because of the quantum "no-cloning" theorem.[4] At a deeper level, QKD resists interception and retransmission by an eavesdropper because in quantum mechanics, in contrast to the classical world, the result of a measurement cannot be thought of as revealing a "possessed value" of a quantum state. A unique aspect of quantum cryptography is that Heisenberg's uncertainty principle ensures that if Eve attempts to intercept and measure Alice's quantum transmissions, her activities must produce an irreversible change in the quantum states (she "collapses the wavefunction") that are retransmitted to Bob. These changes will introduce an anomalously high error rate in the transmissions between Alice and Bob, allowing them to detect the attempted eavesdropping. In particular, from the observed error rate Alice and Bob can put an upper bound on any partial knowledge that an eavesdropper may have acquired by monitoring their transmissions. This bound allows the intended users to apply conventional information theoretic techniques by public discussion to distill an error-free, secret key.

Because it has the ultimate security assurance of a law of Nature, quantum cryptography offers potentially attractive "ease of use" advantages over conventional key distribution schemes: (1) it avoids the "insider threat" because key material does not exist before the quantum transmissions take place, (2) it replaces cumbersome conventional key distribution methods whose security is based on the physical security of the distribution process, and (3) it provides a secure alternative to key distribution schemes based on public key cryptography, which are potentially vulnerable to algorithmic advances and improved computing techniques. Thus, quantum key distribution enables "encrypted communications on demand," because it allows key generation at transmission time over an unsecured optical communications link.

The first quantum key distribution protocol was published by Charles Bennett and Gilles Brassard in 1984 and is now known as "BB84." [5] A further advance in theoretical quantum cryptography took place in 1991 when Ekert proposed[6] that Einstein-PodolskyRosen (EPR) "entangled" two-particle states could be used to implement a quantum cryptography protocol whose security was based on Bell's inequalities. Starting in 1989, 
Bennett, Brassard and collaborators performed the first experimental demonstration of $\mathrm{QKD}$ by constructing a working prototype system for the $\mathrm{BB} 84$ protocol, using polarized photons.[7] Although the propagation distance was only about $30 \mathrm{~cm}$, this experiment is in several ways still the most thorough demonstration of quantum cryptography.

Potentially practical applications of QKD, outside the carefully controlled environment of a physics laboratory, are largely determined by the physics of singlephoton production, the requirement of faithful transmission of the quantum states involved, the existence of high-efficiency single-photon detectors at the required wavelengths, and the compatibility of QKD with existing optical communications infrastructures. In 1992 Bennett published a "minimal" $\mathrm{QKD}$ scheme ("B92") and proposed that it could be implemented using single-photon interference with photons propagating for long distances over optical fibers.[8] Since then, several experimental groups $[9,10,11,12]$ have developed optical fiber-based QKD systems. For example, at Los Alamos we have demonstrated the feasibility of low-error rate $\mathrm{QKD}$ over underground optical fibers that were installed for network applications.[12] We have previously demonstrated QKD over $24 \mathrm{~km}$ of fiber[13] and have operated for over one year at a world-record propagation distance of $48 \mathrm{~km}$.

In recent years there have been considerable developments in the use of free-space laser communications[14] for high-bandwidth terrestrial, surface-to-satellite, satellite-tosatellite and (potentially) deep-space communications. The optical pointing, acquisition and tracking techniques developed for laser communications could be used to make $\mathrm{QKD}$ possible over line-of-sight transmissions in free-space [15],[Publication 1, Publication 2], provided that signal-to-noise and bit rates adequate for cryptographic applications can be achieved. There are certain key distribution problems for which free-space QKD would have definite practical advantages. We believe that free-space QKD could be used for key generation between a low-earth orbit satellite and a ground station [Publication 2] as well as in other applications where laser communications are possible. To demonstrate this possibility we have developed an experimental free-space QKD system. During the first year of this project we demonstrated free-space QKD over a 205-m indoor optical path under ambient (fluorescent) lighting conditions. In the second year we achieved a recordsetting transmission distance of $1-\mathrm{km}$ over an outdoors folded path (to a reflector and back) at night.[Publications 2, 3, 4, 5, 6] In the final year of the project, we performed the first demonstration of free-space $\mathrm{QKD}$ over a point-to-point $0.5-\mathrm{km}$ outdoors path in daylight.[Publication 7] This record-setting distance was limited only by length of the available test range. Because most the optical effects of atmospheric turbulence on a surface-to-space path occur within $2 \mathrm{~km}$ of the ground, our results provide strong evidence 
that cryptographic key material could be generated on demand between a ground station and a satellite (or between two satellites), allowing a satellite to be securely re-keyed on orbit.

\section{Importance to LANL's Science and Technology Base and National R\&D Needs}

Cryptanalysis techniques and algorithms are advancing rapidly and will present increasing information security challenges for orbital assets. It is therefore important to develop new key distribution technologies for use in future generations of satellites. The development of $\mathrm{QKD}$ for satellite communications would represent a major step forward in both security and convenience, allowing a satellite to be securely re-keyed on orbit. With quantum cryptography there is no prior record of a key and no possibility of undetected hostile interception, making the entire key distribution process both simpler and more assured.

In contrast to conventional cryptographic methods whose security is based on assumptions of computational complexity, $\mathrm{QKD}$ is a physics-based technique and as such needs to be experimentally validated. This project has contributed to new Laboratory capabilities in photonics, atmospheric optical physics, quantum information, space physics and the fielding of complex experiments. With the capabilities developed by this project, the Laboratory can provide essential physics input to the intelligence community to ensure the security of future generations of space assets.

\section{Scientific Approach and Accomplishments}

To understand QKD we must first move away from the traditional key distribution metaphor of Alice sending particular key data to Bob. Instead, we should have in mind a more symmetrical starting point, in which Alice and Bob initially generate their own, independent random binary sequences, containing more numbers than they need for the key material that they will ultimately share. Through public discussion they agree on a QKD protocol by which they can perform a bitwise comparison of their sequences using a quantum transmission (over a "quantum channel") and a public discussion of the results (over an authenticated public channel) to distill a shared, random subsequence, which will become the key material. It is important to appreciate that they do not need to identify all of their shared numbers, or even particular ones, because the only requirements on the key material are that the numbers should be secret and random. Several QKD protocols have been developed, but for simplicity we shall describe the minimal $\mathrm{B} 92 \mathrm{QKD}$ protocol[8] in terms of the preparation and measurement of single-photon polarization states. (Cryptographically, the BB84 protocol has certain advantages, but the physics issues involved are identical with B92.) 
In the $\mathrm{B} 92 \mathrm{QKD}$ protocol Alice can produce photons with either of two nonorthogonal polarizations: $\mathrm{V}$ or $+45^{\circ}$ (say); and Bob can make either of two complementary non-orthogonal polarization measurements: $-45^{\circ}$ or $\mathrm{H}$ (say). Alice and Bob generate their own independent sequences of random binary numbers. Next, they proceed through their sequences bit-by-bit in synchronization, with Alice preparing a polarized photon for each of her bits according to the rules:

$$
\begin{gathered}
" 0 " \leftrightarrow V \\
" 1 " \leftrightarrow+45^{\circ}
\end{gathered}
$$

Alice sends each photon over a "quantum channel" to Bob. (The quantum channel is a transmission medium that isolates the quantum state from interactions with the "environment.") Bob makes a polarization measurement on each photon he receives, according to the value of his bit as given by:

$$
\begin{gathered}
\text { "0" } \leftrightarrow-45^{\circ} \\
\text { "1" } \leftrightarrow H
\end{gathered}
$$

and records the result ("pass" $=\mathrm{Y}$, "fail" $=\mathrm{N}$ ). Note that Bob will never record a "pass" (a false positive) if his bit is different from Alice's (they have crossed polarizers). He only records a "pass" on $50 \%$ of the bits that they have in common. In the example of four bits shown in Figure 1 we see that for the first and fourth bits Alice and Bob had different bit values, so that Bob's result is a definite "fail" in each case. However, for the second and third bits, Alice and Bob have the same bit values and the protocol is such that there is a probability of 0.5 that Bob's result will be a "pass" in each case. Of course, we cannot predict in any particular experiment, which one will be a "pass," but in this example the second bit was a "fail" and the third bit was a "pass."

To complete the protocol Bob sends a copy of his ( $\mathrm{Y}$ or $\mathrm{N}$ ) results to Alice, but not the measurement that he made on each bit. (It is at this data-reconciliation stage that the initial key material is required for authentication. This key material can be replaced by a portion of the key material generated by QKD.) He may send this information over a conventional (public) channel, which may be subject to eavesdropping. Now Alice and Bob retain only those bits for which Bob's result was "Y" and these bits become the shared key material. (In the example of Figure 1 the third bit becomes the first bit of the shared key.) An ideal B92 procedure distills on average one shared bit from every four initial bits 
assuming that there are no photon losses in transmission or detection. The $25 \%$ efficiency, $\eta_{\mathrm{Q}}$, of the idealized QKD process is the price that Alice and Bob must pay for secrecy. In a practical system, additional losses in transmission (efficiency factor $\eta_{\mathrm{T}}$ ) and detection (efficiency factor $\eta_{D}$ ) will occur (and can be tolerated). However, these losses only affect the bit rate, not the security.

In a practical system there will be errors in the reconciled data arising from optical imperfections and detector noise, which must be removed before the key material can be used. Alice and Bob can remove these errors using conventional error correcting codes over their public channel, but at the expense of revealing some (parity) information about the resulting key material to Eve. Errors and information leakage will also occur if Eve performs her own measurement of Alice's states on the quantum channel and fabricates new photons to send on to Bob. To take an extreme case, if Eve measures each of Alice's photons using Alice's basis she will introduce a 25\% error rate into Alice and Bob's key material, while correctly identifying $75 \%$ of Alice's bits. Of course, Alice and Bob could readily detect such a large error rate and would not then use their reconciled data for key material, but the eavesdropper could still gain some information at the expense of a proportionately smaller error rate if she only measures a fraction of Alice's photons. It is the goal of quantum cryptography for Alice and Bob to translate an observed error rate into an upper bound on Eve's knowledge of their reconciled data.[16] Such bounds have been established for eavesdropping attacks on individual bits[17] and are the subject of current research in the case of coherent attacks on multiple bits. Error correction should then be followed by a further stage of "privacy amplification" to reduce any partial knowledge acquired by Eve to less than one bit of the final key string.[18] For example, Alice and Bob could choose the parities of random subsets of their error corrected data so that Eve will be forced to have less than one bit of information about the resulting key. These additional stages are performed over the public channel.

Authentication of the public channel transmissions is necessary to avoid a "man-inthe-middle" attack, in which Eve could gain control of both the quantum and public channels, allowing her to masquerade as Bob to Alice and vice-versa. Alice and Bob would then unknowingly generate independent keys with Eve who could use these keys to read all of their subsequent encrypted communications. Alice and Bob need a short, secret authentication key to start the QKD procedure, and can replenish this key with a small portion of the QKD material generated. For authentication based on random hashing they will need $O\left(\log _{2} n\right)$ secret authentication bits for every $n$-bit public transmission.[19] 
So from the foregoing, we see that a QKD procedure may be broken down into the following seven stages:

1. Alice and Bob acquire a secret authentication key.

2. Alice and Bob generate independent secret sequences of random bits.

3. Alice and Bob use the quantum transmissions of a $\mathrm{QKD}$ protocol to compare their sequences and the classical transmissions to identify a random subsequence of shared secret bits.

4. Alice and Bob perform an error correction procedure on the data.

5. Alice and Bob assess (from the error rate) how much knowledge Eve may have acquired.

6. Alice and Bob perform an appropriate privacy amplification procedure over the public channel.

7. Part of the resulting key material is used to replenish the authentication bits required in step 1 , so that the system is ready for the next key generation session.

The result of these steps is a shared, error-free secret key. (It has been proposed that the key bits generated by $\mathrm{QKD}$ should be used for the encryption of communications using the unbreakable "one-time pad" method.[20] However, the key material could equally well, and more practically, be used by Alice and Bob in any other symmetric key cryptosystem.) Of the steps above, only one (step 3 ) involves the experimental physics issues that will be crucial to the practical feasibility of QKD. In our work we have therefore focussed our efforts on this component of QKD. A fully functional key generation system would include careful implementation of the other steps, but these (with the exception of step 5) are better understood and may be readily incorporated once step 3 has been adequately demonstrated. Step 5 relates to the physics of eavesdropping and a full treatment of this topic is beyond the scope of this report. We will therefore limit ourselves to a few additional remarks on this subject.

In the simple form described above, the $\mathrm{B} 92$ protocol is vulnerable to Eve measuring Alice's photons in Bob's basis and only sending on those photons she can identify. (A "Bob's basis" attack.) This will cause a factor of four reduction in bit rate unless Eve sends out multiple photons instead of just one. Alice and Bob can protect against this type of attack if Bob is able to detect the photon number of the received bits, as in our system described below. They could also avoid this problem entirely by using the BB84 protocol, which uses four states instead of two. However, from the perspective of 
the physics, the B92 and BB84 protocols are so similar that BB84 will also be possible under conditions for which QKD with the $\mathrm{B} 92$ protocol is feasible.

The success of QKD over free-space optical paths depends on the transmission and detection of single optical photons against a high background through a turbulent medium. Although these are challenging problems, they can be overcome with careful choices of experimental parameters and the use of various optical techniques developed for laser communications. The atmosphere has a high transmission "window" for light with a wavelength in the vicinity of $770 \mathrm{~nm}$. Photons can be readily produced at this wavelength with rugged, low-power semiconductor lasers and their polarization properties controlled with off-the-shelf optical components. Furthermore, commercial single-photon counting modules (SPCMs) are now available that can count such photons with efficiencies as high as $\eta_{\mathrm{D}} \sim 65 \%$ at rates of up to $1 \mathrm{MHz}$, with dark count rates as low as $50 \mathrm{~Hz}$. The atmosphere is essentially non-birefringent at these wavelengths and so will allow the faithful transmission of the QKD polarization states. However, atmospheric turbulence will introduce both photon arrival time jitter and beam wander (through variations in refractive index). The slow turbulence time-scales involved $(0.1 \mathrm{~s}$ to $0.01 \mathrm{~s})$ allow the jitter to be compensated by transmitting a bright timing laser pulse (which carries no key information) at a different wavelength a short time (100 ns, say) before each QKD photon. The arrival of this bright pulse at the receiver allows a definite timing window to be imposed for the single QKD photon's arrival, because the atmospheric transmission time will not have changed over the intervening short interval. Beam wander caused by atmospheric turbulence reduces the QKD bit rate, but as we will see later is not a critical limitation on surface-to-satellite paths even if left uncontrolled. However, active beam steering ("tip-tilt" control) methods have been developed for laser communications to keep the beam directed onto the receiver. For example, by monitoring a reflected component of the bright timing pulse, an error signal can be derived and fed back to a beam-steering mechanism.

At first sight a more serious concern is that the large background of photons from the sun (or even the moon at night) could swamp the single-photon QKD signal. However, as we will see below, a combination of (sub)-nanosecond timing, narrow wavelength filters $[21,22]$ and a small solid angle for photon acceptance (spatial filtering) at the receiver[Publication 1] can render this background tractable.

Our experimental free-space QKD system has evolved through three stages. In the first year we focused on demonstrating the possibility of free-space QKD without the complications of atmospheric turbulence or high background conditions. We operated over 
a long (205-m) indoor path under ambient lighting conditions. [Publication 1] In the second year we developed a system with which to tackle the problems of outdoors operation but under the restrictions of nighttime operations, for which the atmospheric turbulence and background issues are less demanding than in daylight. For simplicity we operated over a folded path, achieving a record-setting transmission distance of $1 \mathrm{~km}$ that was only limited by the length of the available range.[Publications 2, 3, 4, 5, 6] Finally, in the third year we developed a new system with which to attack the more challenging case of point-to-point, daylight operation. We again achieved a record-setting transmission distance of $0.5 \mathrm{~km}$, limited only by the length of the available range, and we report these results here.[Publication 7]

The QKD transmitter ("Alice") in our system contains a 1-MHz clock that synchronizes the various events. (See Figure 2.) On each "tick" of the clock a 1-ns optical "bright pulse" is produced from a "timing-pulse" laser operating at a wavelength of $\sim 768 \mathrm{~nm}$. After a 100-ns delay, one of two temperature-controlled "data" diode lasers emits a $\sim 1$-ns optical pulse that is attenuated to the single-photon level and constrained by interference filters to a wavelength of $773.0 \pm 0.5 \mathrm{~nm}$. The polarization of the optical pulse from each laser is set to one of the two non-orthogonal settings required for the B92 protocol. The choice of which data laser fires is determined by a random bit value that is obtained by discriminating electrical noise. The random bit value is indexed by the clock tick and recorded in a computer control system's memory. All three optical pulse paths are combined (using beam splitters, BS), directed into a single-mode (SM) optical fiber for delivery to a transmitting telescope, and emitted towards Bob's receiver. The process is then repeated one microsecond later with the next random bit, and so on.

At Bob's QKD receiver the light pulses are collected by a 3.5-inch diameter Cassegrain telescope and directed into a polarization analysis and detection system. (See Figure 3.) The bright pulse triggers an avalanche photodiode detector, and this event sets up an electronic timing "window" about 5-ns long in which a QKD optical data pulse is expected. After emerging from the collection telescope, an optical data pulse encounters an optical beam splitter at which a single-photon would be either transmitted or reflected with equal probabilities. We use this quantum-mechanically random behavior at the beam splitter to perform Bob's random choice of which B92 polarization measurement is made on the arriving optical data pulse. Along the transmitted path, an optical data pulse's polarization is analyzed according to Bob's B92 " 0 " value, while along the reflected path a measurement for $H$-polarization is made using a polarizing beams plitter (PBS). (The PBS transmits $H$-but reflects $V$-polarization.) After each polarization analysis stage, optical 
data pulses pass through interference filters matched to those in the transmitter, and are collected into (spatial filtering) multi-mode optical fibers for delivery to single-photon counting modules (SPCMs), one for each bit value. Of course, for many of the arriving bright pulses there will be no corresponding single-photon detection owing to the efficiency of the B92 protocol, the attenuation experienced by the optical data pulses, and the SPCM's detection efficiency. For events on which one of the two SPCMs triggers, Bob can assign a bit value to Alice's transmitted bit. He records these detected bits in the memory of a computer control system, indexed by the "bright pulse" clock tick. Subsequently, Bob's computer control system transmits a file of index values (but not the corresponding bit values) to Alice over a wireless Ethernet link. Alice and Bob then use those detected bits as the raw bit sequences from which an error-free, secret key is distilled using further communications over the Ethernet channel.

The QKD system was operated for several days over a $0.5-\mathrm{km}$ horizontal outdoors atmospheric range from west (transmitter) to east (receiver) under daylight and nighttime conditions (see Figures 4 and 5). A typical sample of 256 bits identified from 50,000 initial bits under daylight conditions on November 19, 1998 at 4.30pm, is shown in Figure 6 , with Alice's bit value marked as " $A$ " and Bob's as " $B$ ".

The data in Figure 6 contains 5 errors (marked in bold type) and the bit error rate (BER) observed in the whole data set was approximately $1.6 \%$. This would be regarded as unacceptably high in any conventional telecommunications application, but can be tolerated in QKD because of the secrecy of the bits. The effectiveness of our precise timing, wavelength and spatial filtering techniques for mitigating daylight background photon events is shown by the measured background rate of 1 event per 50,000 detector triggers, contributing only approximately $0.4 \%$ to the BER. Detector dark noise makes an even smaller contribution of approximately $0.1 \%$ to the BER. We conclude that the dominant contribution to the BER is from optical misalignment and imperfections of the polarizing elements.

Clearly, errors must be removed before the bit strings can be used as key material. An efficient, interactive error correction procedure has been invented that can remove all errors from such data sets, with BERs of up to 15\%.[23] However, for simplicity in our system we perform a two-dimensional block-parity error correction procedure over the Ethernet channel, which requires Alice to reveal some parity data about the bit strings. An eavesdropper could combine this information with any knowledge acquired through eavesdropping on the quantum transmissions. There are two ways of dealing with this issue. Alice and Bob could encrypt the parity information, which would require them to have more secret bits initially, or they could perform additional privacy amplification to 
compensate for the information revealed, which would produce a shorter key string. We perform a rudimentary privacy amplification procedure by dropping bits from the diagonals of each matrix of data bits. A fully functional QKD system would incorporate a more sophisticated privacy amplification procedure.

The $\sim 5-\mathrm{kHz}$ key rate is adequate for the one-time pad encryption of small image files that we have incorporated into our software control system. (See Figure 7.) Because the one-time pad method requires as many key bits as message bits, the key rate would not be adequate for more lengthy transmissions. This key rate would be acceptable and better used for generating session keys for use in other symmetric key cryptosystems because such keys need only be a few hundred bits in length.

The average photon number per optical data pulse for this data set was $\sim 0.3$, giving a probability of $22 \%$ that the pulse contains exactly one photon, and a probability of $25.9 \%$ that a pulse contains at least one photon. Thus, approximately $15 \%$ of the detectable pulses contain more than one photon. (Such multi-photon pulses can trigger both of Bob's SPCMs, but the rate for these "dual fire" errors is reduced below the key rate by the product of the BER and the multi-photon emission probability. We observed no dual fires in the entire $50-\mathrm{k}$ bit sequence leading to the data in Figure 4. By using four detectors and monitoring the dual-fire rate, Alice and Bob could protect against the "Bob's basis" attack outlined in Section 2. So, a full security analysis of our system must take into account the possibility of Eve performing a beam splitting attack to "tap off" the occasional photon from two-photon pulses. (See Publication [2] for an analysis of this type of attack.)

Our proof-of-concept QKD demonstrations over horizontal terrestrial paths provide strong evidence that surface-to-satellite QKD will be possible. This is because the optical influence of turbulence is the major hurdle to be overcome in surface-to-satellite QKD, and the turbulent effects occur predominantly within the lowest $2 \mathrm{~km}$ of the atmosphere. Ground-to-satellite, satellite-to-ground and satellite-to-satellite QKD should all be possible, for both low-earth orbit (LEO) and geostationary satellites . For illustration we will here estimate the key generation capability of QKD between a ground station and a LEO satellite ( $\sim 300 \mathrm{~km}$ altitude) in one overhead pass (duration $\sim 8$ minutes). Our objective will be to produce multiple new cryptovariables, each of several hundred bits in length. We will assume that the QKD transmitter (Alice) is at the ground station and the receiver (Bob) is on the satellite. (Similar arguments support the viability of satellite-to-ground QKD transmissions, which would have key rate and hardware advantages.)

We have designed our QKD system to operate at a wavelength near $770 \mathrm{~nm}$ where the atmospheric transmission from surface to space can be as high as $80 \%$. Furthermore, at optical wavelengths the polarized QKD photons can be faithfully transmitted because the 
depolarizing effects of Faraday rotation in the ionosphere are negligible. Because the atmosphere is only weakly dispersive, a bright timing pulse (which carries no key information) of $\sim 100$-ps duration can be used to set a short time window ( $\sim 1 \mathrm{~ns}$ ) within which to look for the QKD photon. A single QKD-photon arriving 100 ns after the bright pulse would find that the satellite had moved by less than $1 \mathrm{~mm}$.

To estimate the rate at which QKD photons would be detected at the satellite from the ground station transmitter, we assume $20-\mathrm{cm}$ diameter optics at both the transmitter and satellite receiver, leading to a $\sim 1$-m diameter diffraction-limited spot size at a $300-\mathrm{km}$ altitude satellite. However, there will be beam wander owing to atmospheric turbulence, which at night in a high desert location such as Los Alamos can be 1 to 5 arc seconds [24]. For this analysis we assume a worst case "seeing" of $\sim 10$ times the diffraction limit (i.e. 10 arc seconds of wander) so that the photon collection efficiency at the satellite is $\sim 10^{-4}$. Thus, with a laser pulse rate of $10 \mathrm{MHz}$, one photon-per-pulse on average, an atmospheric transmission of $\sim 80 \%$, a $65 \%$ detector efficiency and allowing for the $25 \%$ intrinsic efficiency of the $\mathrm{B} 92 \mathrm{QKD}$ protocol, a key generation rate of $\sim 250 \mathrm{~Hz}$ is feasible. (There would be a factor of two higher key rate with the BB84 protocol.)

Higher key rates would be possible under more typical seeing conditions. Also, with a simple beam tilt feedback system, as used in laser communications systems, the beam could be locked onto the satellite, increasing the key rate to $\sim 40 \mathrm{kHz}$. A retroreflector on the satellite would return a portion of each bright pulse to the transmitter with a $\sim 2$ ms delay, which is much shorter than the time-scale of atmospheric turbulence fluctuations. (From the ground, the satellite would move through an angle of only $~ 50$ micro radians in this time.) It would also be possible to place the QKD transmitter on the satellite and the receiver on the ground. Because most of the optical influence of atmospheric turbulence would occur in the final $\sim 2-\mathrm{km}$ of the beam path, a higher key rate would then be possible even without tilt control.

To determine if this key rate is useful we must also consider the error rate. We first consider errors arising from background photons arriving at the satellite on a nighttime orbit with a full moon and under (poor) 10-arc second seeing conditions. A typical radiance observed at the satellite at the transmission wavelength would then be $\sim 1 \mathrm{~mW} \mathrm{\textrm {m } ^ { - 2 }}$ $\mathrm{str}^{-1} \mu \mathrm{m}^{-1}$ or $\sim 4 \times 10^{15}$ photons $\mathrm{s}^{-1} \mathrm{~m}^{-2} \mathrm{str}^{-1} \mu \mathrm{m}^{-1}$. We will assume that the receiver "sees" a solid angle $\sim$ five times the apparent size of the source (i.e. 5 arc seconds) and that there is a 1-nm bandwidth interference filter placed in front of the detector, giving a background photon arrival rate of $\sim 225 \mathrm{~Hz}$ (full moon). (For comparison, detector dark counts would be $\sim 50 \mathrm{~Hz}$.) However, the single-photon detector would only be triggered by precursor 
bright pulses impinging on the satellite, giving a detector trigger rate of $\sim 90 \mathrm{kHz}$ (without beam tilt control). With a 1-ns time window applied to the detector, the (fractional) bit error rate (BER) from background photons would therefore be $\sim 5 \times 10^{-5}$ (full moon. With beam tilt control the fractional BER from background photons would be $\sim 4 \times 10^{-5}$. In practice, errors from optical component limitations and misalignments will be larger, amounting to a 1 to 2 percent BER based on our experience.

From this simple analysis using worst-case estimates, we see that $\mathrm{QKD}$ between a ground station and a low-earth orbit satellite should be possible on nighttime orbits. During the several minutes that a satellite would be in view of the ground station there would be adequate time to acquire the satellite, perform the QKD transmissions for $\sim 1$ minute, and produce a minimum of $\sim 10,000$ raw bits, from which a shorter error-free key stream of several thousand bits would be produced after error correction and privacy amplification. Under more typical seeing conditions or with beam tilt control implemented, up to $10^{5}$ key bits could be produced in the 1-minute QKD transmission. A cryptographically useful quantity of key material could therefore be generated between a ground station and a LEO or geostationary satellite using available technology. (Satellite to satellite $\mathrm{QKD}$ transmissions would also be possible.)

On daytime orbits the background radiance would be $\sim 4,000$ times larger $\left(\sim 2 \times 10^{19}\right.$ photons s$\left.~^{-1} \mathrm{~m}^{-2} \mathrm{str}^{-1} \mu \mathrm{m}^{-1}\right)$ than under a full moon, but a narrow atomic vapor filter ( $\sim 0^{-2} \mathrm{~nm}$ filter width) [25] would keep the background photon arrival rate to only $\sim 10 \mathrm{kHz}$. Assuming a typical daytime seeing of 10 arc seconds [24], the key rate would be $\sim 250 \mathrm{~Hz}$, and the BER from background photons would then be $\sim 2 \times 10^{-3}$ (without tilt control). QKD is therefore also likely to be possible on daytime orbits.

Our results are the first practical demonstration that point-to-point free-space QKD is feasible under daylight conditions outside a laboratory, achieving a realistic propagation distance of $0.5 \mathrm{~km}$ that was only limited by the length of the test range. We are now in the process of improving the system and anticipate performing a 2-km daylight demonstration early in 1999, possibly increasing to $7 \mathrm{~km}$ later. Free-space QKD could therefore be used in conjunction with terrestrial laser communications systems that are now commercially available. Our results also provide strong evidence that cryptographic key material could be generated on demand between a ground station and a satellite (or between two satellites) using $\mathrm{QKD}$, allowing a satellite to be securely re-keyed on orbit.

The development of $\mathrm{QKD}$ for satellite communications would represent a major step forward in both security and convenience. In contrast to conventional key distribution methods whose security is based on assumptions of computational complexity, QKD is a physics-based technique and as such needs to be experimentally validated under the 
conditions of its intended use. To our knowledge the primary physics requirements for this application of $\mathrm{QKD}$, namely the transmission and detection of single photons between a ground station and an orbital asset, have never been demonstrated. However, many of the optical acquisition, pointing, tracking and adaptive optics techniques developed for laser communications with satellites can be directly applied to this problem. Therefore, we believe that a surface-to-satellite $\mathrm{QKD}$ demonstration experiment would be a logical and realistic next step in the development of this new field. Furthermore, we believe that the development of $\mathrm{QKD}$ for re-keying of satellites on-orbit will be essential as an alternative to traditional key distribution methods that can potentially become vulnerable to unanticipated algorithmic or computational advances.

\section{Publications}

1. Buttler, W. T. et al., Free space quantum key distribution, Phys Rev A57, 2379 (1998).

2. Buttler, W. T. et al., Practical free-space quantum key distribution over $1 \mathrm{~km}$, Phys Rev Lett 81, 3283 (1998).

3. Buttler, W. T. et al., Free-space quantum key distribution at night, Proc. SPIE 3385, 14 (1998).

4. Hughes, R. J. et al., Practical free-space quantum key distribution, to appear in the proceedings of the first NASA QCQC conference, Palm Springs, CA, February 1998.

5. Hughes, R. J. et al., Free-space quantum cryptography, LA-UR-98-3962, invited paper to appear in the Proceedings of "Quantum Communications and Measurement" Evanston, IL, August 23-27, 1998.

6. Hughes, R. J. et al., Quantum cryptography for secure free-space communications, to appear in the proceedings of the SPIE Photonics West conference, San Jose, CA, January 1999.

7. Hughes, R. J. et al., Practical quantum cryptography for secure free-space communications, submitted to the CRYPTO'99 conference, Santa Barbara, CA (August 1999). 


\section{References}

[1] D. Atkins et al., "The Magic Words are Squeamish Ossifrage," Advances in Cryptology-ASIACRYPT '94 Proceedings, Springer-Verlag, 1995, pp.263; Cowie, J., et al. "A world wide number field sieve factoring record: on to 512 bits," pp. 382-394 in: K. Kim and T. Matsumoto (eds), Advances in Cryptology - Asiacrypt '96, Lecture Notes in Computer Science \# 1163, Springer-Verlag, Berlin, 1996.

[2] For reviews see Hughes, R. J. et al., "Quantum Cryptography," Contemporary Physics 36, 149 (1995); Bennett, C. H. et al., "Quantum Cryptography," Scientific American 257 no.10, 50 (1992).

[3] Clauser, J. F., Phys Rev D 9, 853 (1974); Grangier, P. et al., Euro Phys Lett 1, 173 (1986).

[4] Wooters, W. K. and Zurek W. H., Nature 299, 802 (1982).

[5] Bennett, C. H. and Brassard, G., "Quantum Cryptography: Public Key Distribution and Coin Tossing," Proceedings of IEEE International Conference on Computers, Systems and Signal Processing, Bangalore (New York, IEEE, 1984).

[6] Ekert, A. K., "Quantum Cryptography Based on Bell's Theorem," Phys. Rev. Lett. 67, 661 (1991).

[7] Bennett, C. H. and Brassard, G., "The Dawn of a New Era for Quantum Cryptography: The Experimental Prototype is Working," SIGACT NEWS 20, no. 4, 78 (1989); Bennett, C. H. et al., "Experimental Quantum Cryptography," J. Crypto. 5, 3 (1992).

[8] Bennett, C. H., "Quantum Cryptography Using Any Two Non-Orthogonal States," Phys. Rev. Lett. 68, 3121 (1992).

[9] Townsend, P. D., Rarity, J. G. and Tapster, P., "Single Photon Interference in $10 \mathrm{~km}$ Long Optical Fiber Interferometer," Elec. Lett. 29, 634 (1994); Townsend, P. D., "Secure Key Distribution Based on Quantum Cryptography," Elec. Lett. 30, 809 (1994); Marand, C. and Townsend, P. D., "Quantum Key Distribution Over Distances as Long as 30 km," Opt Lett. 20, 1695 (1995); Townsend, P. D., Nature 385, 47 (1997).

[10] Muller, A. et al., "Experimental Demonstration of Quantum Cryptography Using Polarized Photons in Optical Fibre Over More Than $1 \mathrm{~km}$," Europhys. Lett. 23, 383 (1993); Muller, A. et al., "Quantum Cryptography Over $23 \mathrm{~km}$ in Installed Under-lake Telecom Fibre," Europhys. Lett. 33, 335 (1996).

[11] Franson, J. D. and Ilves, H., "Quantum Cryptography Using Optical Fibers," Appl. Optics 33, 2949 (1994).

[12] Hughes, R. J. et al., "Quantum cryptography over underground optical fibers," Lecture Notes in Computer Science 1109, 329 (1996).

[13] Hughes, R. J. et al., "Secure Communications Using Quantum Cryptography," PROC SPIE 3076, 2 (1997).

[14] See, for example, Gagliardi, R. M. and Karp, S., Optical Communications (Wiley, New York, 1995)

[15] Jacobs, B. C. and Franson, J. D., Opt. Lett. 21, 1845 (1996).

[16] Lutkenhaus, N., "Estimates for practical quantum cryptography," (1998).

[17] Ekert, A. K. et al., Phys Rev A 50, 1047 (1994).

[18] Bennett, C. H. et al., "Generalized Privacy Amplification," IEEE Trans. Inf. Theory 41, 1915 (1995).

[19] Wegman, M. N. and Carter, J. L., "New hash functions and their use in authentication and set equality," J. Comp. Sys. Sci., 22, 265-279 (1981).

[20]Vernam, G. S., Trans. Am. IEE 45, 295 (1926).

[21] Walker, J. G. et al., Quant. Opt. 1, 75 (1989).

[22] Seward, S. F. et al., Quant. Opt. 3, 201 (1991).

[23] Brassard, G. and Salvail, L., "Secret-key reconciliation by public discussion," (1994).

[24] Wilson, K. E. et al., Proc. SPIE 2990, 23 (1997).

[25] Zhilin, H., Sun, X., and Zeng, X., Opt. Communications 101, 175 (1993). 


\begin{tabular}{|l|l|l|l|l|}
\hline Alice's bit value & 1 & 0 & 1 & 0 \\
\hline Alice's polarization & $+45^{\circ}$ & $\mathrm{V}$ & $+45^{\circ}$ & $\mathrm{V}$ \\
\hline Bob's polarization & $-45^{\circ}$ & $-45^{\circ}$ & $\mathrm{H}$ & $\mathrm{H}$ \\
\hline Bob's bit value & 0 & 0 & 1 & 1 \\
\hline Bob's results & $\mathrm{N}$ & $\mathrm{N}$ & $\mathrm{Y}$ & $\mathrm{N}$ \\
\hline
\end{tabular}

Figure 1. A four-bit example of B92 quantum key distribution.

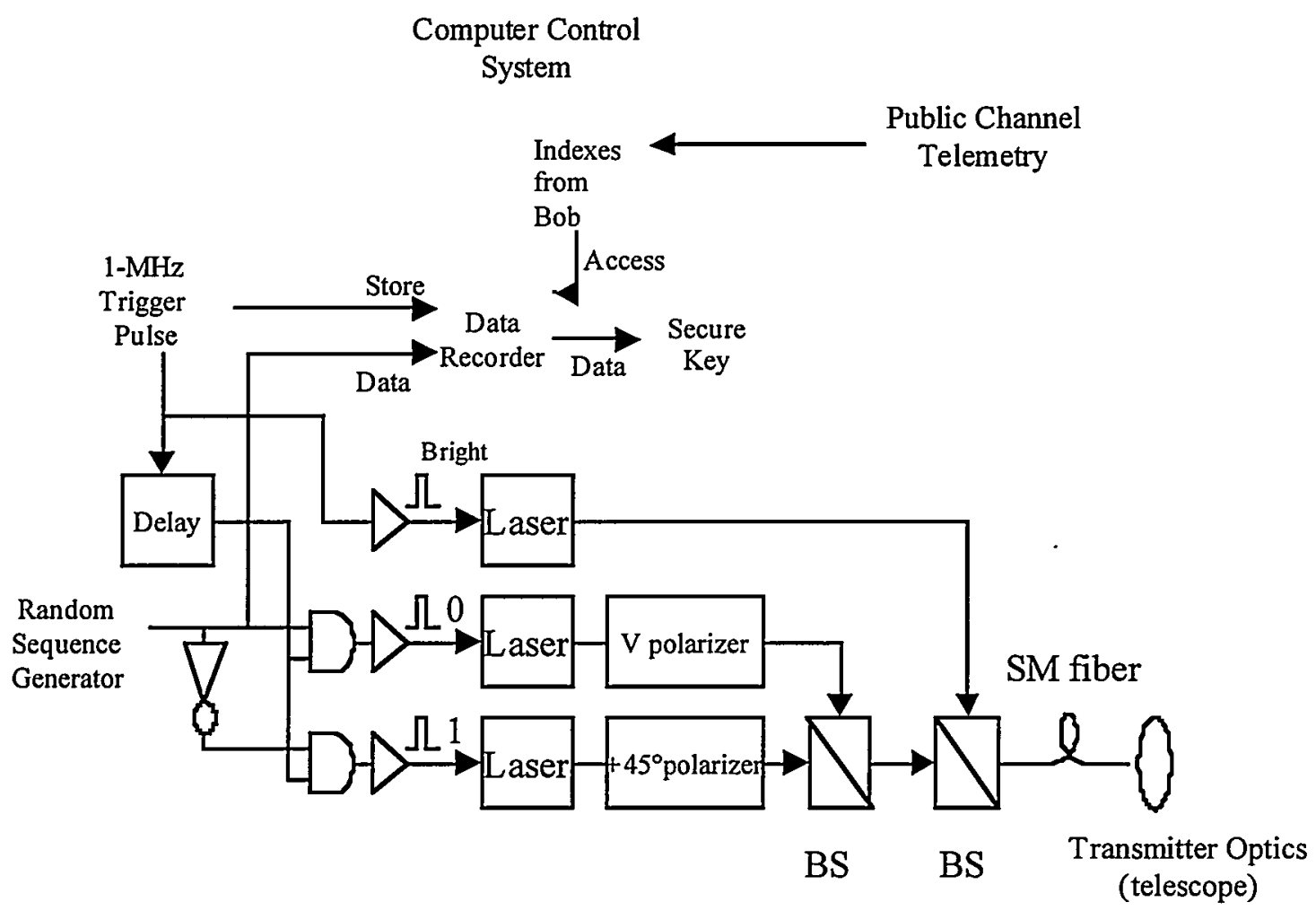

Figure 2. QKD transmitter ("Alice") block diagram. 


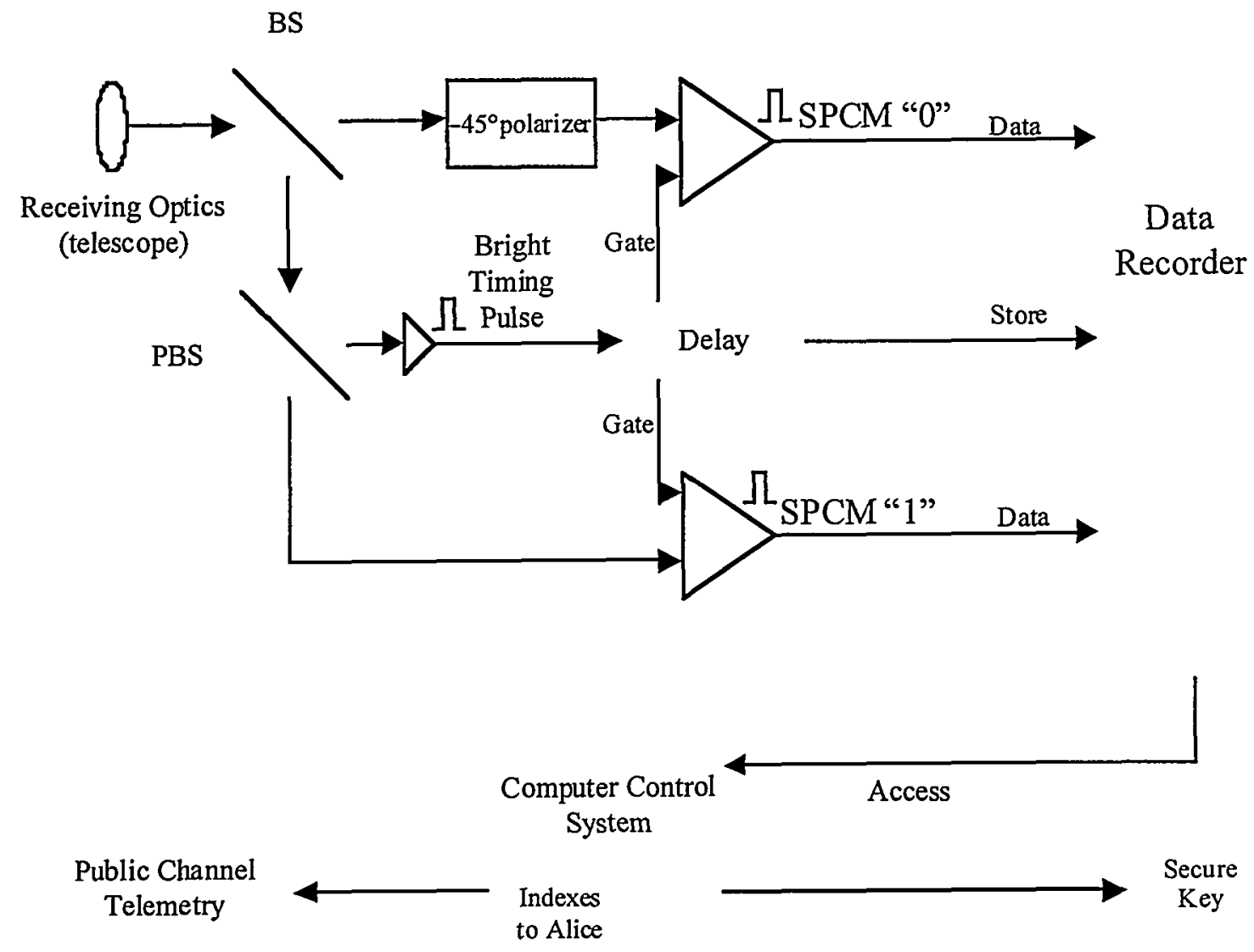

Figure 3. QKD receiver ("Bob") block diagram. 


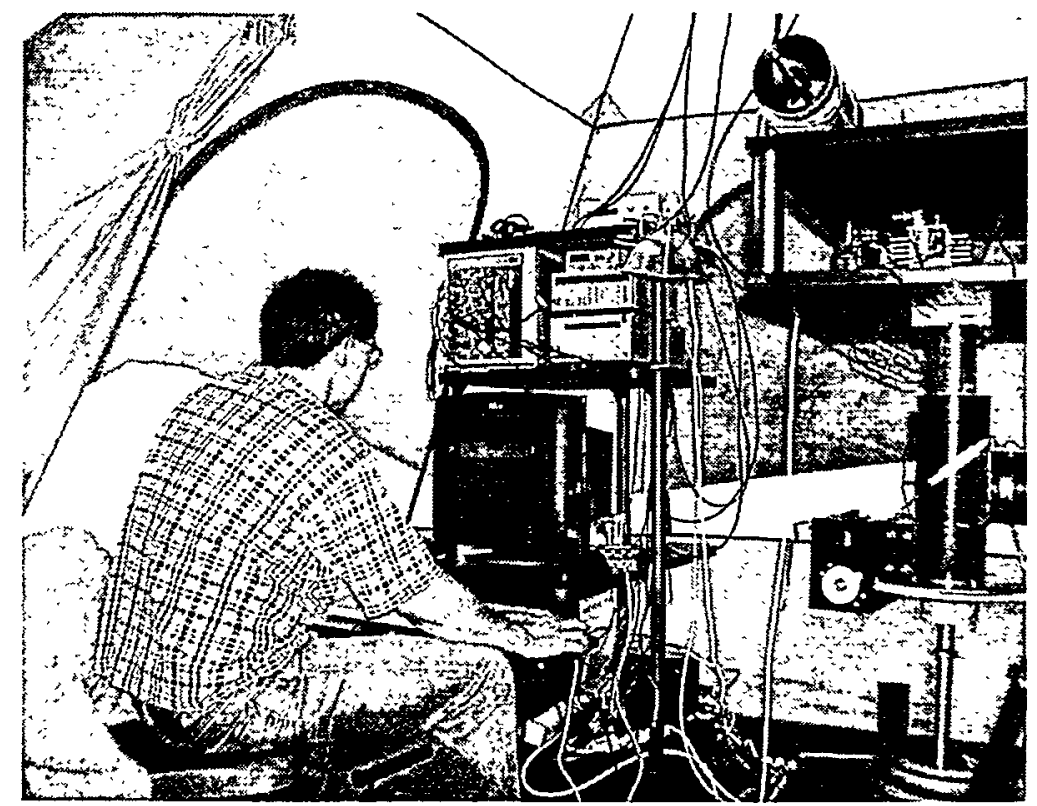

Figure 4. Operation of the free-space QKD transmitter.

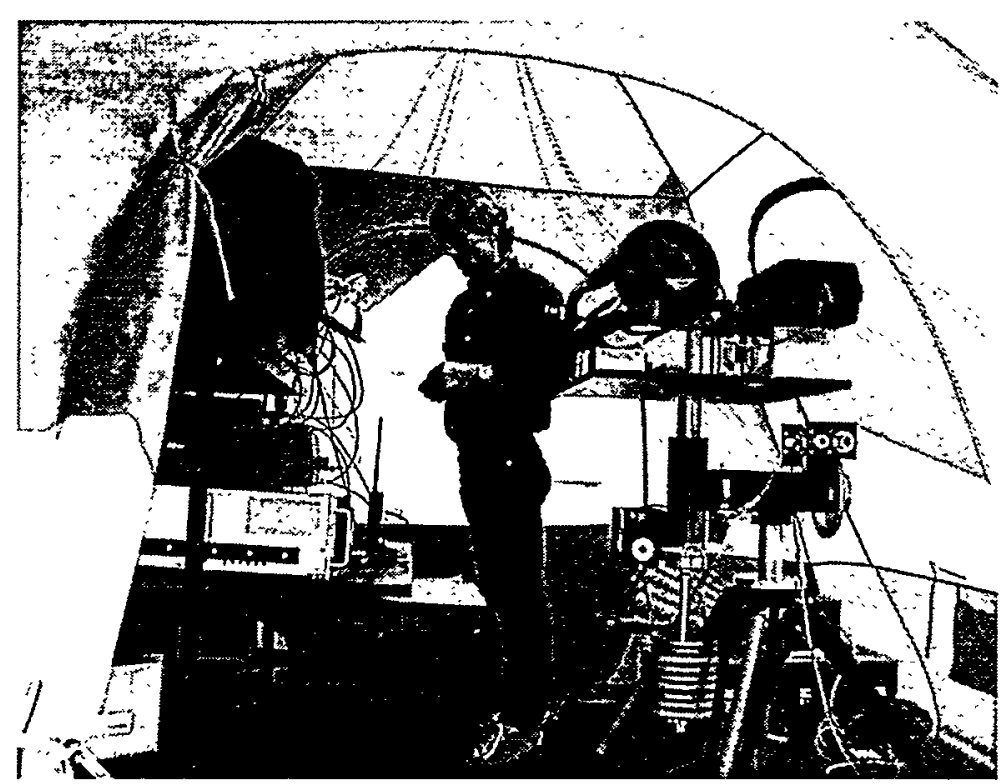

Figure 5. The free-space QKD receiver in operation. 
A 1010111010011001100011110000110110011011101100111001100110000001 B 1010111010011001100011111000110110011011101100111001100110100001

A 1100111001101010100101111000111010110000110011101110110110110011 B 1100111001101010100101111000111010110000110011101110110110110011

A 0100000001010001000100000001001001000111000100111100100001001001 B 0100000101010001000100000001001001000111000100111100100001011001

\section{A 0111010110110010011100101110111100101101000101011001111100101111 B 1111010110110010011100101110111100101101000101011001111100101111}

Figure 6. A raw sample of bits identified by Alice and Bob as shared using the B92 protocol over a $0.5-\mathrm{km}$ daylight path. Alice's bit value is marked as " $A$ " and Bob's as "B."

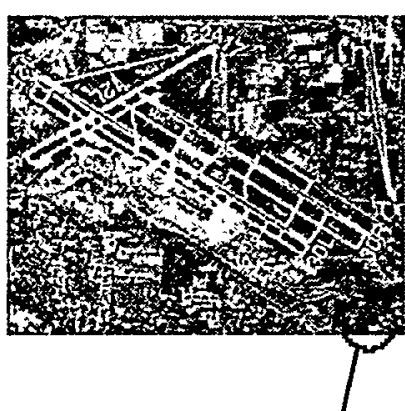

Encryption

by adding a word of key

to each pixel

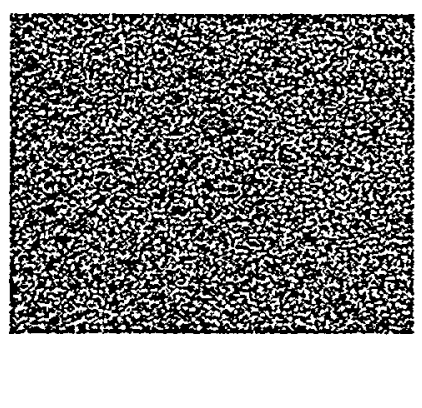

Encrypted Image

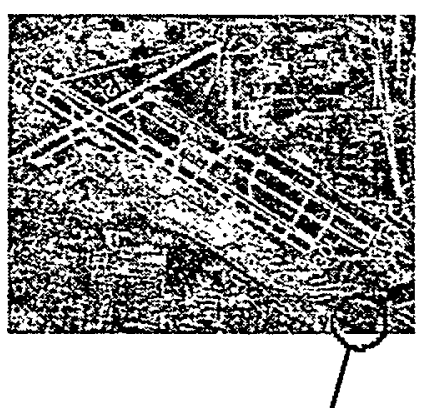

Decryption

by subtracting a word of key

from each pixel

Figure 7. Encryption and decryption of an aerial image of St. Louis airport using the "one-time pad" method with key material produced by free-space quantum cryptography. In this example, the error correction was turned off to show the effects of bit errors in the key on the decrypted image. 